\title{
SARS-CoV-2 and Portal Vein Thrombosis: A Rare Gastrointestinal Manifestation of COVID-19
}

\author{
Sindhura Kolli ${ }^{1}$, Veeral M. Oza ${ }^{2}$ \\ 1. Internal Medicine, New York University Langone Hospital, New York, USA 2. Gastroenterology, University of South \\ Carolina-Greenville, Greenville, USA
}

Corresponding author: Sindhura Kolli, sindhura.kolli@gmail.com

\begin{abstract}
Portal vein thrombosis is defined as a clot within the trunk or intrahepatic branches of the portal vein. Sequelae involves either partial or complete recanalization. However, in patients with liver disease, it can progress to a cavernoma instead of recanalization. This can result in gastrointestinal bleeding and intestinal infarction. Its rising incidence in severe acute respiratory syndrome coronavirus 2 is an important clinical aspect that needs to be addressed and treated.
\end{abstract}

Review began 03/27/2021 Review ended 04/06/2021 Published 04/07/2021

\section{() Copyright 2021}

Kolli et al. This is an open access article distributed under the terms of the Creative Commons Attribution License CC-BY 4.0., which permits unrestricted use, distribution, and reproduction in any medium, provided the original author and source are credited.
Categories: Internal Medicine, Gastroenterology, Infectious Disease

Keywords: portal vein thrombosis, pvt, sars-cov-2, gastroenterology, covid-19

\section{Introduction}

Coagulopathy in severe acute respiratory syndrome coronavirus 2 (SARS-CoV-2) or coronavirus disease 2019 (COVID-19) was a disturbing discovery as the incidence of micro- and macrovasculature thrombi increased in proportion to the rising number of SARS-CoV-2 cases. While accounts of deep vein thrombosis (DVT) and pulmonary emboli were regularly added to the COVID-19 literature, only a handful incidences of portal vein thrombosis (PVT) have been reported thus far [1]. This rare but prevalent gastrointestinal effect of COVID19 should be recognized as a possible etiology of abdominal pain for early recognition and treatment to prevent complications and further sequelae.

\section{Case Presentation}

A 44-year-old female patient with no past medical history presented to the emergency department (ED) with abdominal pain, abdominal bloating, and chest discomfort. Abdominal pain in the epigastric and right upper quadrants was dull, aching, and was present for one to two weeks prior to admission. It was accompanied by bloating and no alleviating factors. She visited her primary care physician, who performed a right upper quadrant ultrasound that was concerning for a PVT and sent her to the hospital. She denied any calf pain, recent surgery, immobilization, or any other risk factors for a possible DVT. PVT was confirmed in the ED using a computed tomography (CT) (Figure 1).

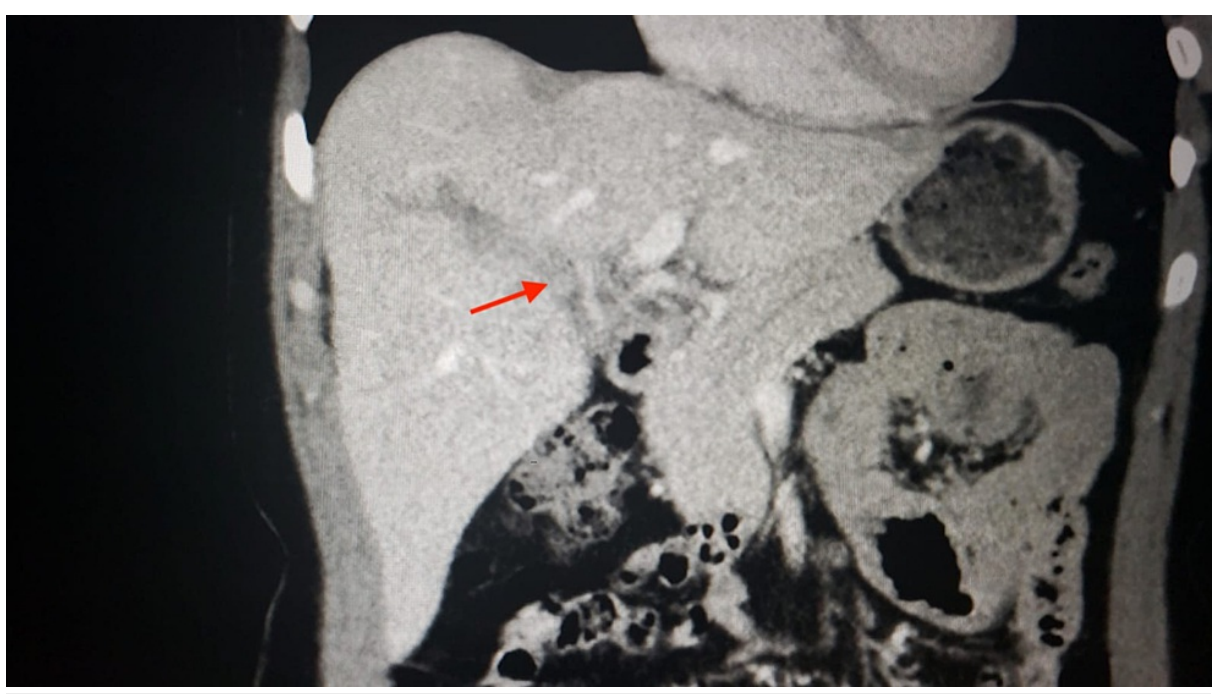

FIGURE 1: CT imaging of PVT in a COVID-19 positive patient.

CT, computed tomography; PVT, portal vein thrombosis; COVID-19, coronavirus disease 2019 
Her vitals were within normal limits and stable. Her labs, including her hepatic workup, were also unremarkable. She was placed on a therapeutic heparin drip and coumadin. Hypercoagulable workup outside of prothrombin time, activated partial thromboplastin time, fibrinogen, and platelet count included lupus anticoagulant panels; activated protein $\mathrm{C}$ resistance, protein $\mathrm{C}$, and protein $\mathrm{S}$ activity; and antithrombin activity were negative. Specific coagulation factor levels were not indicated as preliminary workup was negative. CT of her abdomen did not demonstrate any other provoking factors, including cirrhosis, for a thrombosis. She was subsequently discharged on coumadin for economic reasons. Her COVID-19 test performed during her admission returned positive after she was discharged. Upon discharge, she also developed a dry cough as a late presentation of her COVID-19. She was counseled on a vitamin Kappropriate diet as well as common medication interactions with coumadin and scheduled for outpatient follow-up post appropriate quarantine.

\section{Discussion}

The commonly reported gastrointestinal manifestations of COVID-19 include nausea, vomiting, diarrhea, abdominal pain, and to a lesser extent abnormal liver function tests (LFTs). Abnormal LFTs could be secondary to sepsis-related inflammation or direct viral-associated hepatocellular assault through entry of the SARS-CoV-2 virus via ACE-2 receptors expressed on hepatocytes and hepatic cholangiocytes. Similarly, the inflammatory state incitation of a cytokine storm, complement activation, and coagulation cascade is thought to create a pro-coagulopathic state, leading to a higher incidence of venous thrombosis and, less commonly, arterial thrombosis. Both gastrointestinal manifestations and the presence of venous or arterial thromboembolism bear poorer outcomes in COVID-19 patients [2,3].

PVT is not a surprising sequelae given it often occurs against the backdrop of an inflammatory state, such as cirrhosis, or when a prothrombotic predisposing condition is identified. PVT presents with nonspecific symptoms such as abdominal pain, fever, small-volume abdominal ascites, and splenomegaly [4]. However, when complications such as intestinal ischemia, bowel infarction, and ileus arise, the mortality rate plummets from an $85 \%$ five-year survival rate to $20-50 \%$ rapidly $[5,6]$. Labs demonstrate normal LFTs, except for an elevated alkaline phosphatase if biliary pathology occurs, with prolonged prothrombin time, decreased coagulation factors, and an increase in D-dimer [7]. Ultrasound is the initial imaging preference with a sensitivity of $80-100 \%$ and specificity of $88-98 \%$. CT and magnetic resonance imaging are preferred if complications are suspected as they bestow further illumination regarding nearby organ infarction or thrombus extension [8]. Treatment involves anticoagulation with heparin or low-molecular-weight heparin or unfractionated heparin for three to six months based on assessment of risk factors both from COVID-19 severity and underlying medical conditions, probability of portal vein recanalization, and presence of PVT complications $[3,8]$.

\section{Conclusions}

Given how rarely PVT has been occurring in the setting of COVID-19 and its nonspecific presentation, it can be easily misdiagnosed or overlooked. However, given its devastating sequelae, it should be considered a differential during the investigation of gastrointestinal manifestations of the widely sweeping and highly prevalent SARS-CoV-2.

\section{Additional Information \\ Disclosures}

Human subjects: Consent was obtained or waived by all participants in this study. Brooklyn Hospital Center issued approval 4156682. Thank you for submitting the Publication materials for this project. Brooklyn Hospital IRB has APPROVED your submission. No further action on this submission is required at this time. Conflicts of interest: In compliance with the ICMJE uniform disclosure form, all authors declare the following: Payment/services info: All authors have declared that no financial support was received from any organization for the submitted work. Financial relationships: All authors have declared that they have no financial relationships at present or within the previous three years with any organizations that might have an interest in the submitted work. Other relationships: All authors have declared that there are no other relationships or activities that could appear to have influenced the submitted work.

\section{References}

1. La Mura V, Artoni A, Martinelli I, et al.: Acute portal vein thrombosis in SARS-CoV-2 infection: a case report. Am J Gastroenterol. 2020, 115:1140-1142. 10.14309/ajg.0000000000000711

2. Sultan S, Altayar O, Siddique SM, et al.: AGA institute rapid review of the gastrointestinal and liver manifestations of COVID-19, meta-analysis of international data, and recommendations for the consultative management of patients with COVID-19. Gastroenterology. 2020, 159:320-334. 10.1053/j.gastro.2020.05.001

3. Abou-Ismail MY, Diamond A, Kapoor S, Arafah Y, Nayak L: The hypercoagulable state in COVID-19: incidence, pathophysiology, and management. Thromb Res. 2020, 194:101-115. 10.1016/j.thromres.2020.06.029

4. Plessier A, Darwish-Murad S, Hernandez-Guerra M, et al.: Acute portal vein thrombosis unrelated to cirrhosis: a prospective multicenter follow-up study. Hepatology. 2010, 51:210-218. 10.1002/hep.23259 


\section{Cureus}

5. Condat B, Pessione F, Helene Denninger M, Hillaire S, Valla D: Recent portal or mesenteric venous thrombosis: increased recognition and frequent recanalization on anticoagulant therapy. Hepatology. 2000, 32:466-470. 10.1053/jhep.2000.16597

6. Kumar S, Sarr MG, Kamath PS: Mesenteric venous thrombosis. N Engl J Med. 2001, 345:1683-1688.

10.1056/NEJMra010076

7. Condat B, Valla D: Nonmalignant portal vein thrombosis in adults . Nat Clin Pract Gastroenterol Hepatol. 2006, 3:505-515. 10.1038/ncpgasthep0577

8. Chawla YK, Bodh V: Portal vein thrombosis. J Clin Exp Hepatol. 2015, 5:22-40. 10.1016/j.jceh.2014.12.008 